\title{
Towards Automated Segmentation Methods for 3D Tomography Studies of the Morphology of Carbon Nanoglobules in Chondritic Meteorites
}

\author{
K. J. Yakal-Kremski ${ }^{1,2}$, N. D. Bassim², K. Scott ${ }^{3}$, and R. M. Stroud ${ }^{2}$ \\ 1. NRC-NRL Cooperative Fellow, 4555 Overlook Ave. SW, Washington, DC 20375, USA. \\ 2. Naval Research Laboratory, Code 6366, 4555 Overlook Ave. SW, Washington, DC 20375, USA. \\ ${ }^{3 .}$ National Institute of Standards and Technology, Gaithersburg, MD 20899, USA.
}

The presence of nano-scale, roughly spherical carbonaceous features has been shown in several samples of chondritic meteorites [1-4]. There have been a few proposed mechanisms by which these carbon nanoglobules may form [2,5]. Quantification of nanoglobule prevalence, morphology, and relationship to other meteorite components can provide important evidence to elucidate how the nanoglobules form, and how the carbon contained therein may be altered by interactions with fluids and nearby minerals present on the asteroid from which the meteorite originated.

It has recently been shown that 3D FIB-SEM tomography can be utilized to collect reconstruction volumes of chondritic meteorite grains, as was done on a grain from the Tagish Lake 5b sample [6]. A representative FIB-SEM cross-section is shown in Figure 1, showing the complex nature of the sample, as well as highlighting the selected nanoglobule regions of interest. Segmentation of the nanoglobules for the data set in [6] was achieved manually, which poses a significant challenge to further study of this and other samples, as it is very time consuming and subject to variation in the resultant output based on what individual is carrying out the segmentation.

Several challenges arise when trying to formulate an efficient and effective automated method for segmentation of the nanoglobules and other constituent phases present in a chondritic meteorite particles. The porous nature and irregular geometries of the particles make segmentation of the entire volume challenging, as back-contrast from the far wall of pores makes automated identification difficult, and regions of which with similar contrast may be misidentified as the nanoglobules. Carbon epoxy infiltration, a common method for filling pores to even contrast, is not a viable method, as the nanoglobules are also carbonaceous, and would not be easily distinguishable from the background epoxy. Additionally, there are other carbon phases, large carbonaceous veins called clasts, which have similar contrast but need to be segmented as a separate phase. While not related to the segmentation of the nanoglobules per se, the sheer number of present phases is also daunting, which is reflected in the complex image histogram given in Figure 2. Identification of nearby phases may be important in determining how the nanoglobules may interact with the parent body $[1,7]$.

Despite the complex nature of the problem, an automated, or pseudo-automated, method for segmentation is desired, and will be attempted for the Tagish Lake sample. This method should also be portable for use with other meteorite samples or otherwise. An automated segmentation scheme would allow for much higher throughput, and a more regimented and less subjective definition for each of the phases desired for segmentation. This too would allow for further analysis of the Tagish Lake sample reported earlier, as only $\sim 20 \%$ of the total reconstruction volume was analyzed due to the slow manual segmentation. 
References:

[1] C. M. O'D. Alexander, et al., Geochimica et Cosmochimica Acta 71 (2007), p. 4380-4403

[2] L. A. J. Garvie and P. R. Buseck, Earth and Planetary Science Letters 224 (2004), p. 431-439

[3] K. Nakamura, et al., International Journal of Astrobiology 1 (2002), p. 179-189

[4] B. T. De Gregorio, et al., Meteoritics \& Planetary Science 48 (2013), p. 904-928

[5] M. Saito and Y. Kimura, The Astrophysical Journal 703 (2009), p. L147-L151

[6] R. M. Stroud, et al., Proceedings of the 44th Lunar and Planetary Science Conference (2013)

[7] C. D. Herd, et al., Science 332 (2011), p. 1304-1307

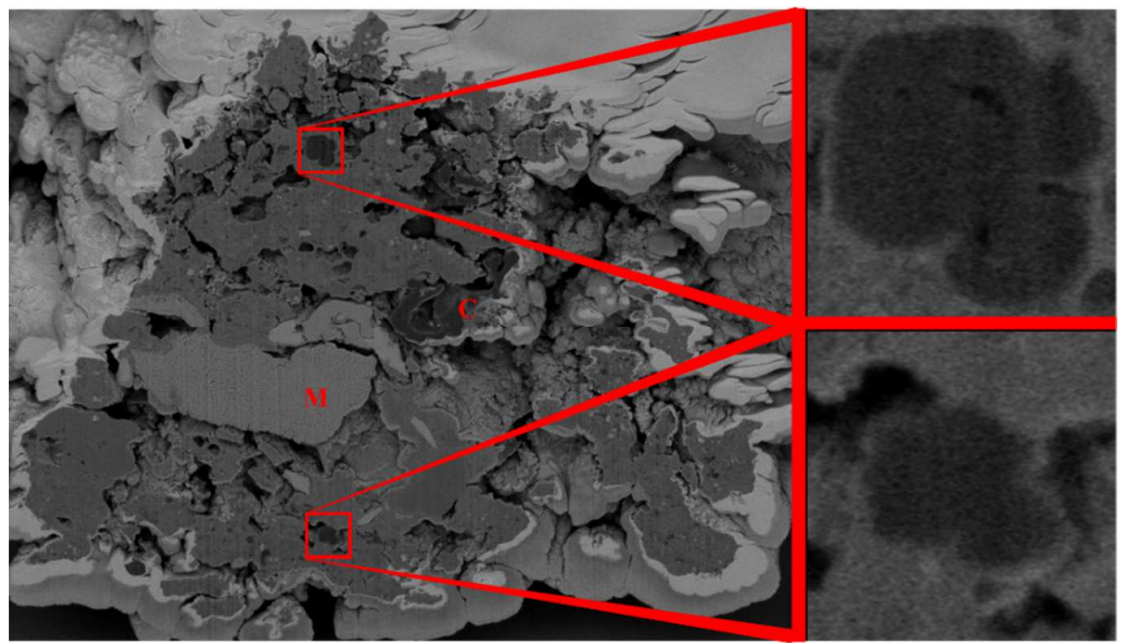

Figure 1. Cross-section of a FIB-SEM collected data set on a grain from a Tagish Lake meteorite sample (left). Image width is $3.1 \mu \mathrm{m}$. Many phases can be observed, including a large carbonaceous clast (C), a spongy magnetite particle (M), unfilled porosity, and many other phases. Insets on right are two nanoglobule regions of interest for identification and segmentation. Inset widths are each 140nm. Note that an internal void is present in the top nanoglobule, whereas the bottom nanoglobule appears dense.

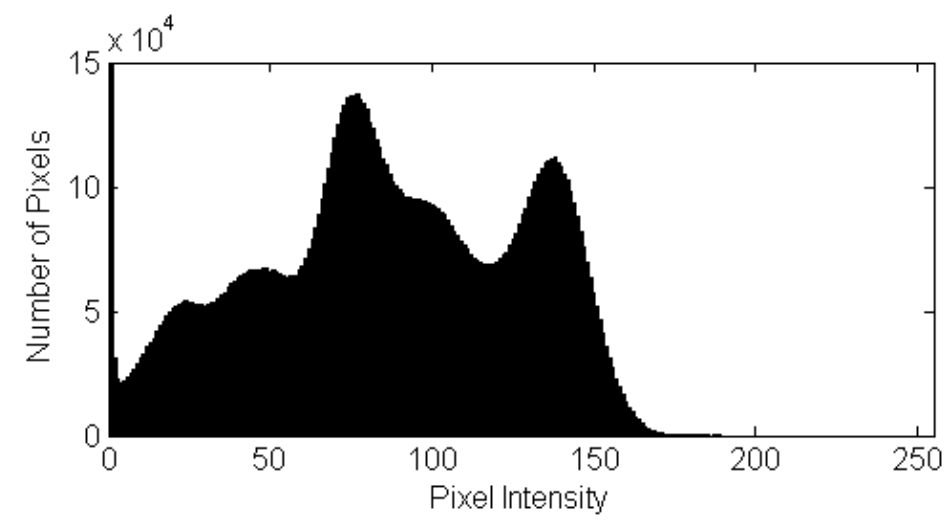

Figure 2. Histogram of a selected interior region of the microstructure cross-section shown in Figure 1. While individual peaks are distinguishable, there is a high extent of peak overlap. This makes simpler segmentation techniques difficult to use, due to high uncertainty of pixel allocation. 